\title{
Mecanismos para la asignación de los recursos financieros a partir de la descentralización en el estado de a alisco
}

\author{
Ricardo Pérez-N úñez, MC, M en $C,{ }^{(1)}$ Armando Arredondo-López, MC, M en C, D r en C, ${ }^{(2)}$ \\ Blanca Pelcastre, Lic en Psic, M en Psic Soc, Dra en Psic Soc. ${ }^{(2)}$
}

\begin{abstract}
Pérez-Núñez R,Arredondo-López A, Pelcastre B. Mecanismos para la asignación de los recursos financieros a partir de la descentralización en el estado de Jalisco. Salud Publica Mex 2006;48:127-140.
\end{abstract}

\section{Resumen}

Objetivo. Analizar, a partir de la perspectiva de los tomadores de decisiones, el proceso de asignación de los recursos financieros en los servicios de salud del estado de Jalisco (SS)), México, en el contexto de la descentralización. Material y métodos Mediante una aproximación cualitativa, con entrevistas semiestructuradas a informantes clave de puestos directivos como técnica de levantamiento de información, se documentó, a través del análisis del discurso de los informantes, la experiencia de los SSJ en materia de asignación de recursos financieros. De septiembre a noviembre de 2003, se exploró la percepción de directivos y administrado res so bre el grado de auto no mía que tienen en la toma de decisiones y el proceso que se sigue en la asignación de los recursos financieros, para identificar los criterios que se utilizan y sus justificaciones. Resultados. Desde el punto de vista de los tomadores de decisiones, a partir de la descentralización se ha incrementado la autonomía de los SS), aunque el grado de decisión permanece limitado debido, principalmente, al gran gasto administrativo asociado a nómina. En este sentido, las implicaciones imputables a las condiciones laborales aún no descentralizadas son todavía evidentes. En los SSJ se han establecido sistemas propios e innovadores para la asignación de los recursos financieros por regiones sanitarias y hospitales, con base en incentivos administrativo-gerenciales y en productividad, asimismo realizando ajustes por grado de marginación y rezago poblacional, bajo el criterio de equidad. Conclusiones Las condiciones generales de trabajo y el poder de decisión
Pérez-Núñez R,Arredondo-López A, Pelcastre B. Mechanisms for allocating financial resources after decentralization in the State of Jalisco. Salud Publica Mex 2006;48:127-140.

\begin{abstract}
A bstract
Objective.To analyze, from the decision maker's perspective, the financial resource allocation process of the health services of the state of Jalisco (SS), per its abbreviation in spanish), within the context of decentralization. Material and Methods Through a qualitative approximation using semi-structured individual interviews of key personnel in managerial positions as the method for compiling information, the experience of the SSJ in financial resource allocation was documented. From September to N ovember 2003, the perception of managers and administrators regarding their level of autonomy in decision-making was explored as well as the process they follow for the allocation of financial resources, in order to identify the criteria they use and their justifications. Results From the point of view of decision-makers, autonomy of the SSJ has increased considerably since decentralization was implemented, although the degree of decision-making freedom remains limited due mainly to high adminstrative costs associated with salaries. In this sense, the implications attributable to labor situations that are still centralized are evident. Some innovative systems for financial resource allocation have been established in the SSJ for the sanitary regions and hospitals based upon administrative-managerial and productivity incentives. Adjustments were also made for degree of marginalization and population lag, under the equity criterion. Conclusions General work conditions and decision-making auto nomy of the sanitary regions constitute outstanding aspects pending decentralization. Although decentralization has granted more
\end{abstract}

(1) Instituto Nacional de Salud Pública/Escuela de Salud Pública de México. Cuernavaca, Morelos, México.

(2) Centro de Investigación en Sistemas de Salud, Instituto N acional de Salud Pública. Cuernavaca, Morelos, México.

Fecha de recibido: 24 de enero de 2005 • Fecha de aprobado: 3 de noviembre de 2005

Solicitud de sobretiros: M en C. Ricardo Pérez N úñez, Centro de Investigación en Sistemas de Salud, IN SP. A venida Universidad \# 655, colonia Santa María A huacatitlán 62508, Cuernavaca, Morelos, México.

Correo electrónico: rperez@ correo.insp.mx 
asignado a las regiones sanitarias constituyen un aspecto pendiente de descentralizar. Si bien la descentralización ha otorgado mayor autonomía a los SS], el nivel de decisión para la asignación de recursos financieros se ha concentrado en los niveles jerárquicos más altos.

Palabras clave: asignación de recursos financieros; descentralización; México autonomy to the SS, the level of decision-making freedom for allocating financial resources has been held within the highest hierarchical levels.

Keywords: financial resources allocation; decentralization; Mexico
A partir del informe del Banco Mundial de 1993, se ha llamado la atención sobre la cuestión del financiamiento, haciendo hincapié en cuatro problemas principales: a) la distribución de los recursos hacia intervenciones costo-inefectivas; b) la inequidad en el acceso a servicios básicos; c) la ineficiencia en la utilización de los recursos disponibles, y d) los costos crecientes de la atención en salud que hacen que el gasto agregado del sector crezca a un ritmo superior al del ingreso. ${ }^{1} \mathrm{El}$ incremento de los costos de los servicios de salud y, en consecuencia, la necesidad de aumento del gasto y cambios en los métodos de asignación de recursos, han suscitado múltiples inquietudes en los tomadores de decisiones, sobre todo en los planificadores y ejecutores de las políticas públicas. De ahí que se vuelva impostergable la necesidad de aplicar nuevos métodos de asignación de recursos fundamentados en criterios técnicos y no sólo basados en la tendencia histórica del gasto en salud -que es lo que se ha venido haciendo en los países de América Latina. ${ }^{2,3}$

En México, la descentralización se debió a varias causas: la escasez de recursos precipitada por las crisis económicas; la pérdida persistente del poder local; la carencia efectiva de participación popular en la gestión estatal; la limitada transparencia de los procesos del aparato estatal y, finalmente, la incapacidad de la política social para hacer frente a la crisis, así como para fomentar la toma de decisiones en el lugar mismo donde se generan los problemas y las necesidades. ${ }^{4-6}$ El estado de Jalisco fue una de las 14 entidades pioneras en descentralizarse en la década de los $80,6,7$ proceso que interrumpió la crisis económica de $1986 .{ }^{5}$ En 1995, el gobierno federal retomó la descentralización, incluyéndola como uno de los principales lineamientos del Programa de Reforma del Sector Salud 1995-2000, con el objeto de "incrementar la eficiencia de la atención a la población abierta". ${ }^{8}$ A pesar de que durante muchos años la Secretaría de Salud (SSA) definió las partidas del gasto en salud para todo el país, su objetivo era que, en adelante, las entidades federativas decidieran totalmente el destino de los recursos federales que re- cibían, siempre y cuando cumplieran, al menos, un mínimo de acciones de salud determinadas en el ámbito federal -el llamado "Paquete Básico de Servicios de Salud"-. El esquema de presupuestación es, por tanto, una pieza clave para modificar la relación entre los prestadores de servicios (los estados) y la autoridad normativa (SSA), la cual pretendía transitar de un esquema de control directo hacia uno de autonomía de gestión, en donde el gobierno federal asumiera la función de evaluar los resultados obtenidos por los operadores del gasto, sin por ello interferir en sus decisiones. ${ }^{9}$

La implantación del proceso en los años 90 comenzó formalmente con la firma, en agosto de 1996, del Acuerdo Nacional para la Descentralización de los Servicios de Salud. ${ }^{10}$ Este último define un conjunto de principios básicos, entre los que destacan el manejo y operación directos de los servicios de salud por parte de los estados federales, así como de los recursos financieros -incluidas las cuotas de recuperación- con la posibilidad de distribuirlos según sus necesidades y prioridades locales. Por otra parte, todas las estructuras administrativas operadas por el gobierno federal se integrarían al ámbito estatal. 9,11 Sin embargo, algunos aspectos, como las condiciones generales de trabajo, permanecieron centralizados. ${ }^{10,11}$

En 1998 la asignación del presupuesto de la SSA hacia los estados pasó a formar parte del Fondo de Aportaciones para los Servicios de Salud (FASSA) del Ramo 33.* Antes de ese año, la distribución regional del presupuesto federal se efectuaba por el Ramo $12, \ddagger$

\footnotetext{
* La asignación de los recursos financieros federales a las distintas instancias gubernamentales se realiza a través de ramos. El Ramo 33, creado en 1998 como una vertiente del federalismo por medio de la adición y reforma a la Ley de Coordinación Fiscal (LCF), corresponde a las aportaciones federales para entidades federativas y municipios.

$\ddagger$ Actualmente el Ramo 12 es el recurso asignado a las instancias gubernamentales directamente implicadas en el combate a la pobreza.
} 
en el cual la SSA, utilizando presupuestos históricos, elaboraba todos los capítulos y conceptos del gasto de cada entidad federativa. No obstante, a partir de 1998 los estados adquirieron una considerable autonomía para llevar a cabo su programación y presupuestación, con amplia libertad para distribuir el gasto federal entre diversos programas. Estas decisiones se toman al interior del organismo público descentralizado de servicios de salud de cada estado dentro de la Junta de Gobierno, integrada por el gobernador del estado respectivo -quien la preside-, algunas secretarías estatales, un representante de la SSA y un representante sindical. Según informes oficiales de la Presidencia, para 1999 las entidades federativas ejercían ya más de $70 \%$ del presupuesto total en salud, mientras que en 1994 ese porcentaje había sido inferior a $25 \% .^{12}$ A raíz de la descentralización, los estados sólo tienen que notificar la aplicación de los recursos federales a los congresos locales, informando a la SSA exclusivamente sobre cuestiones muy generales de manejo presupuestal y sin rendirle cuentas sobre sus decisiones específicas de asignación. ${ }^{9}$

Esta "versión oficial", que ve a la descentralización como un proceso exitoso, contrasta con algunas posiciones que sostienen que la descentralización no se ha llevado hasta sus últimas consecuencias y que, además, eso difícilmente sucederá, debido sobre todo a la falta de voluntad política, el tipo de federalismo que priva en el país y las grandes asimetrías que existen entre los estados. ${ }^{13}$ En este mismo sentido, Cardo$\mathrm{zo}^{9}$ señala algunas limitaciones de la primera etapa de descentralización: el único margen de autonomía financiera concedida a las entidades federativas fue la proporción del gasto solventada con sus propios recursos; los empleados de los servicios estatales de salud continuaron perteneciendo a la nómina federal, aunque los gobiernos de los estados fueron facultados para actuar como patrones sustitutos en cuestiones sindicales y para participar en la administración del personal. ${ }^{9}$ Por otro lado, destacan las enormes restricciones sobre manejo presupuestal y patrimonial impuestas a los estados descentralizados -baja autonomía de gestión- y su gran dependencia respecto a los fondos federales. En este sentido, el aspecto financiero parece haber sido uno de los principales obstáculos para una descentralización más profunda durante la década de los 80 , no sólo por la falta de autonomía en el manejo de los recursos, sino también por la preocupación de los gobernadores de que los recursos federales no bastaran para atender los nuevos compromisos adquiridos. ${ }^{9}$

Pese a las intenciones y objetivos de la política de descentralización, en fechas recientes se ha observado, en estudios de casos llevados a cabo en México y en Latinoamérica, que la descentralización no ha mejorado la eficiencia, la equidad, la calidad y el nivel de satisfacción por parte de los usuarios. Cabe destacar, además, un aumento del número de decisiones inadecuadas: los líderes de las comunidades* tienden a asignar el gasto para satisfacer a grupos de interés o para actividades sanitarias que no son siempre recomendables, como, por ejemplo, gastos en atención médica de alta tecnología en vez de programas preventivos. En raras ocasiones los líderes toman en cuenta la mejora de la salud de la población. La inequidad se ha elevado también: con la descentralización financiera los estados y municipios más pobres tienen menos recursos que los ricos, lo que incrementa la injusticia en la globalidad del país. Particularmente en México, la descentralización ha aumentado el gasto debido a la organización de las nuevas administraciones, a la capacitación del personal descentralizado y del personal central que deben reorientar sus actividades, y a las alzas de salarios causadas por la homologación del personal. Asimismo, existe un incremento del gasto por la pérdida de las economías de escala en la compra de insumos y en contratos para el mantenimiento de equipos, sobre todo en áreas de poca población. Incluso la eficiencia se vio reducida en gran medida por los duelos de poder entre los administradores centrales y administradores provinciales, y por la afectación de los intereses de los trabajadores del sector salud. Así pues, el proceso de descentralización en México se ha caracterizado por una gran improvisación que, sin duda, ha sido en gran parte responsable de los fracasos mencionados. ${ }^{13-16}$

Desde la descentralización, algunos estados han tratado de adoptar nuevos criterios técnicos para una distribución más equitativa de los recursos para programas de salud; empero, no se han evaluado los resultados de la aplicación de nuevos mecanismos de asignación de financiamiento en salud. ${ }^{3}$ En este sentido, es importante determinar los mecanismos y criterios empleados para la asignación de recursos al interior de los estados. Los estudios de caso permiten identificar fortalezas y debilidades para retroalimentar los procesos y generar experiencias que puedan utilizarse en otros contextos. En este estudio, se resalta la perspectiva de los administradores en la identificación del grado de autonomía para la toma de decisiones, evaluada a través de dos dimensiones: el tipo de

\footnotetext{
* Por líderes comunitarios se entiende a los gobiernos municipales o locales y cualquier otra instancia involucrada en la toma de decisiones en materia de salud en el ámbito local, como juntas directivas, patronatos y hasta élites locales o clases dominantes.
} 
políticas y decisiones administrativas relevantes para operar, y el punto hasta el cual las decisiones se toman en el nivel central, ${ }^{17}$ esto es, el grado de decisión, entendido como el porcentaje del presupuesto total en salud que pueden asignar libremente. Una vez conocido el grado de decisión, fue importante identificar los criterios en los que basaban sus decisiones, quién asignaba el presupuesto, cómo se formaban los equipos de análisis y decisión, así como los incentivos empleados -esto es, las recompensas y fuerzas disuasorias a las que se enfrentan $-{ }^{17}$ en los Servicios de Salud del estado de Jalisco.

\section{Material y métodos}

Esta investigación es un estudio de caso que forma parte del macroproyecto "Equidad e indicadores financieros en el contexto de la descentralización: actores políticos y estrategias para la reforma del sector salud (FASE 2)" evaluado y aprobado por los comités de investigación y ética del Instituto Nacional de Salud Pública. En este estudio, llevado a cabo de septiembre a noviembre de 2003 mediante una aproximación cualitativa, se documenta la experiencia de los SSJ en materia de asignación de recursos financieros. Como técnica de levantamiento de información se aplicaron entrevistas semiestructuradas a informantes clave de los SSJ, para lo cual se elaboró una guía basada en los siguientes temas de interés:

- Poder de decisión.

- Grado de decisión.

- Tipo de decisión (criterios y justificaciones).

La población con la que se trabajó fue el personal directivo de los SSJ, entre los cuales se seleccionó una muestra por criterio, de acuerdo con los siguientes perfiles:

- Personal directivo del ámbito estatal cuyas funciones estuvieran directamente relacionadas con la detección de problemas, el establecimiento de prioridades con respecto a las necesidades y la asignación de recursos financieros.

- Personal del ámbito estatal que contara con un mínimo de experiencia de cinco años en actividades de coordinación general u operativa, implantación, supervisión o evaluación de la descentralización.

- Personal del ámbito estatal que contara con un mínimo de experiencia de cinco años en actividades de administración, planeación y/o coordinación financiera de los SSJ.

- Personal que hubiera participado durante la implantación de la primera fase de la descentralización.
Algunas personas seleccionadas para el estudio formaron parte del macroproyecto original "Equidad e indicadores financieros en el contexto de la descentralización: actores políticos y estrategias para la reforma del sector salud (FASE 2)", de tal manera que se analizaron sus entrevistas como información secundaria (4 entrevistas). Estas entrevistas fueron presenciadas por un observador no participante que realizó las anotaciones pertinentes para la reestructuración de la guía de entrevista con la que, posteriormente, se regresó para recabar la información primaria. Se incluyó a los demás participantes seleccionados para fines de este estudio, constituyendo la información primaria (7 entrevistas). A todos ellos se les informó de manera clara y precisa en qué consistía el estudio y, una vez que aceptaron participar en el mismo, se les solicitó su consentimiento informado de manera verbal para grabar las entrevistas. Estas se llevaron a cabo dentro de un marco de confidencialidad, anonimato y respeto, que garantizó en todo momento un espacio de seguridad en donde pudieron expresarse libremente, sin que esto implicara perjuicio futuro para su persona o puesto.

Se hizo un análisis del discurso de las entrevistas con apoyo del programa Atlas-ti versión 4.2. Se delimitaron las categorías de análisis de interés para el estudio y se clasificó la información. Se definieron familias de códigos de información relacionada, para finalmente integrar el informe de resultados a partir de la interpretación y análisis de la información obtenida en las entrevistas. Los resultados se presentan organizados por categorías analíticas.

\section{Resultados}

\section{Poder y grado de decisión}

De acuerdo con el personal entrevistado, los SSJ sí tienen poder de decisión sobre los recursos financieros de que disponen, especialmente sobre aquellos del Ramo 33 que se presentan libres y sin etiquetas. Los participantes reconocieron que la experiencia adquirida en el ejercicio de descentralización de la década de los 80 los preparó mejor desde el punto de vista técnico y les aportó mayores conocimientos y capacidades para el manejo de los recursos financieros. Consideraron, además, que los cambios legales experimentados durante el segundo proceso descentralizador les dan mayor margen de decisión:

Creo en la figura jurídica de descentralización pues precisamente nos abre mucho las puertas en ese sentido, ¿por qué?, porque nos crea como una figura jurídica de organismo público descentralizado, nos da salud pública de méxico / vol.48, no.2, marzo-abril de 2006 
mucho más autonomía en el manejo de los recursos, mucho más oportunidad jurídica en los compromisos y mucho más oportunidad operativa.

En este sentido, en la Junta de Gobierno se reconoce una figura clave para que los SSJ puedan administrar los recursos con relativa autonomía, ya que, como autoridad máxima, aprueba transferencias internas importantes o avala la asignación de recursos adicionales, habilitando, además, a los tomadores de decisiones para que decidan, con relativa libertad, las medidas que tengan lugar, especialmente en caso de urgencias epidemiológicas. En las sesiones de la Junta de Gobierno se identifican, de manera colegiada, las necesidades, la urgencia de las mismas; y el área de planeación propone y decide dónde y cómo se aplicarán los recursos financieros, decisión que es avalada por medio de consenso o mayorías.

Ante ellos (...) presentamos la solicitud (...) para mover dinero que originalmente viene presupuestado en Capítulo 1000, ${ }^{*}$ por ejemplo, en las economías que se lleguen a generar del Capítulo 1000, poderlas gastar en la sustitución del centro de salud de allá de aquella localidad o en la compra de más medicamento; esa autorización te la da una instancia aquí estatal del OPD y no tienes que decirle a México -oye Julio Frenk me das chance de gastar diez millones de pesos que salieron de economía de nómina- ésa es una de las ventajas.

Al interior de la institución, durante el ejercicio anual, se decide en qué gastar los excedentes o de dónde sacar los faltantes -transferencias internas o solicitud de recursos extraordinarios- según el comportamiento del presupuesto, esto es, de acuerdo con los gastos y los disponibles que se tengan. Sin embargo, su libertad para decidir está condicionada a la cantidad de recursos con que cuentan o que finalmente les autorizan. En este sentido, el poco poder que los SSJ tienen ante la Federación cuando se les asignan los recursos, sumado a las recientes disminuciones del gasto operativo, genera un sentimiento de frustración en los tomadores de decisiones, a quienes, en sus propias palabras, les toca "simplemente administrar la miseria".

Por otro lado, coincidieron en reconocer que su poder de decisión está restringido en virtud de los al-

\footnotetext{
* El registro y reporte de gasto se efectúa de acuerdo al clasificador por objeto del gasto para la administración pública. El Capítulo 1000 corresponde a servicios personales: pago de nóminas, estímulos y compensaciones económicas, entre otras.
}

tos gastos relacionados con el Capítulo 1000. Si bien el Ramo 33 les llega en apariencia libre, el hecho de que las condiciones laborales no se hayan descentralizado y que los reglamentos de escalafón y capacitación, el control y los estímulos del personal de base sigan regidos por la normatividad federal, limita considerablemente el grado de autonomía que tienen, sobre todo si se considera que de 70 a 92\% del presupuesto total está relacionado con el Capítulo 1000.

Hay muchas cosas que están muy amarradas en la normatividad federal, la administración del recurso humano nos parece bastante difícil, eso de que se autorizan días económicos hasta 10 días y luego los permisos con goce de sueldo y el pago por una asistencia perfecta, tú sabes, que nos imponen de México, es una desventaja porque de nuestro presupuesto hay que tomar o sea la previsión presupuestal para pagar eso. A nosotros nunca nos preguntaron si la queríamos, eso nos llegó ya derechito, nos afecta nuestro presupuesto.

Otro problema que añaden a este panorama es el pago de cubre-incidencias - pago de personal eventual para cubrir al personal de base que está de permiso o incapacitado, o bien espacios que requieren de personal para el que no hay plazas autorizadas-, mismo que se duplicó entre 2001 y 2002. Todo esto, opinan, lleva a una realidad en la que se está invirtiendo mucho dinero en nómina y administración y poco en gasto de operación para la atención en salud y gasto de inversión, los cuales, incluso, han disminuido de forma paulatina, situación que consideran inadmisible.

Además, todo el recurso relacionado con el Ramo 12 les llega etiquetado por la Federación para los diferentes programas verticales (PAC, Oportunidades, etc.), recurso que debe gastarse en el tiempo y la forma que la SSA determina, de tal manera que no tienen el mínimo poder sobre este recurso.

Por ejemplo el no presupuestar recursos para inversión
que desde México vienen teniendo tan ajustado el gas-
to de operación [...] en la asignación de los irreducti-
bles pues no le transfieres, no puedes mover dinero de
gasto de operación para llevarlo a gasto de inversión o
a compra de equipamiento, sustitución de unidades de
salud que ya están muy viejas y que hay que construir
las nuevas, entonces, bueno de todas estas amarras to-
davía que son hechas desde México pues son las des-
ventajas.

Algunos de estos programas van acompañados de equipamiento y compensaciones salariales asignados directamente por la Federación, situación que, mani- 
fiestan, ha generado inconformidad entre los trabajadores e inequidad laboral dentro de la institución, pues se perciben ingresos y equipo en función del tipo de programa al que están incorporados y no de las actividades, las prioridades estatales o el nivel jerárquico . Por otro lado, existe todo un marco legal y normativo, tanto federal como estatal, según el origen del recurso, que rige la forma en que debe justificarse y gastarse dicho recurso -por ejemplo, compras y adquisiciones-. En cuanto al recurso de origen estatal, gran parte llega a los SSJ preasignado, ya sea por el Legislativo o por el Ejecutivo.

Aunque hubo quienes dijeron desconocer o no recordar el grado de decisión que tiene la institución, el porcentaje del gasto total registrado en los SSJ sobre el que, según los entrevistados, se puede decidir -esto es, el dinero que no viene previamente etiquetadovaría de $40 \%$ a sólo $5 \%$, aunque la libertad para asignar los recursos financieros se restringe en la medida en que se desciende en la escala de jerarquías.

Las direcciones generales tienen poder y autonomía de decisión sobre el dinero que se les asigna, el cual generalmente responde a la programación anual que realizan de acuerdo con las prioridades de cada área, establecidas mediante "negociación" directa con el director general (secretario de Salud), y que sirve como medio de expresión de su propia autonomía. No obstante, el ejercicio del gasto debe ser congruente con los lineamientos y políticas establecidas por la Dirección General de Planeación (DGP), en colaboración con el secretario de Salud, o bien aquellas directrices acordadas en reuniones de trabajo donde participa el personal de más alto rango jerárquico. Las transferencias internas de recursos deben ser aprobadas por la DGP, previa solicitud expresa.

En cuanto a las direcciones de área, se identificaron dos tendencias claras. Por un lado, algunas áreas, que son meramente operativas, no tienen ingerencia administrativa en el manejo del recurso asignado, con un grado de decisión de 0\%. Su programación anual no siempre se respeta y sólo en ocasiones tienen poder de decisión para algunos recursos asignados directamente por la Federación.

De hecho, este año ha sido una experiencia catastrófica al creer que teníamos asignados una cierta cantidad de dinero, de la cual íbamos a hacer uso, y a la hora de la hora me dijeron que había desaparecido ¿sí?, que alguien tomó la decisión de usarla en otras cosas. Entonces, en ese sentido es una dirección de área operativa mas no administrativa ¿sí?, estamos muy limitados en ese sentido. En cuanto al presupuesto otorgado por la Cruzada Nacional hasta en esta última etapa, nos die- ron oportunidad de nosotros tomar decisiones sobre el dinero que la Cruzada Nacional designó hacia Jalisco, sí estamos comenzando a llevar control de los proyectos y el uso de ese recurso.

Por el otro lado, ciertas áreas consideradas aparentemente como más prioritarias, tienen gran poder de decisión y reciben mayores recursos financieros, respetando sus programaciones anuales, con grado de decisión referido de 99 a 100\% -sin contar Capítulo 1000-, y otras de 5\% -considerando el Capítulo 1000-. Se puede decir que no todas las áreas tienen el mismo poder de decisión sobre los muchos o pocos recursos asignados a su cargo y que esto ocurre en función de la percepción de los más altos funcionarios con respecto a las áreas respectivas. Sin embargo, todas las direcciones deben sujetarse a los lineamientos correspondientes; así por ejemplo, el Capítulo 5000* requiere de todo un proceso de licitación y aprobación administrativa.

Se refiere que las regiones sanitarias tienen desconcentrada la facultad de decidir en qué se va a gastar el dinero -distribución del recurso entre programas- de acuerdo con el establecimiento de las prioridades en materia de necesidades que, en conjunto con la DGP, realizan a través de su programación anual, sujetándose a la normatividad y políticas de la DGP e informando quincenalmente del uso que se le da a dicho recurso. Tienen la capacidad de realizar compras pequeñas de material y medicamentos en caso de requerirlo de urgencia, aunque existen conceptos como el Capítulo 5000 para lo que no se les da libertad de decisión, y sólo pueden realizar estos gastos previa autorización por parte de la DGP o la Dirección General de Administración (DGA).

Una idea referida con persistencia fue la importancia que ha cobrado, en años recientes, la cuota de recuperación como estrategia de refinanciamiento e incentivo administrativo, situación que se ha traducido en un incremento del poder que los SSJ tienen sobre los recursos financieros - pues el dinero recaudado es recurso que se puede asignar libremente, según sus propios criterios y prioridades-, así como en mayores captaciones, pues parte del recurso recaudado se queda en el mismo centro de responsabilidad que las registra, y con ello les otorga más poder de decisión. En este sentido, se cita el siguiente ejemplo:

\footnotetext{
* El Capítulo 5000 corresponde a recursos destinados para la adquisición de bienes muebles e inmuebles.
} 
[El] Hospital General de Occidente que es el hospital más grande aquí está adquiriendo equipo vía arrendamiento financiero con el cual puede hacer tomografías computarizadas y otros servicios que antes nosotros mandábamos a que el paciente lo hiciera por fuera, donde le iban a cobrar 2, 3 mil, 4 mil pesos por un estudio de éstos, para que se lo haga en las propias instalaciones y nada más le cobramos mil, por decir algo; con todo y todo, es rentable hacerlo así, es decir, Hospital General de Occidente es capaz de generar el doble de cuotas de recuperación; de lo que se está generando, cobrando ese tipo de servicios, aun pagando lo que hay que pagar por la adquisición de ellos, entonces hicimos una fuente alterna de financiamiento; simplemente, cobrar los servicios que antes no cobrábamos, sino que los hacían otros; [...] nosotros pensamos finalmente que si el $52 \%$ del gasto en salud sale de los bolsillos de los propios pacientes, bueno pues es una buena, excelente fuente de financiamiento porque estamos hablando de mucho más de nuestro presupuesto seguramente.

En general, todo aquello que genere o ahorre recursos incrementa indirectamente el poder y la autonomía de los tomadores de decisiones. Aquí se destacó la labor y liderazgo del secretario de Salud para negociar el reetiquetamiento del recurso federal y la gestión de mayores recursos estatales y federales, así como de financiamiento de instancias nacionales e internacionales. Mencionaron, como ejemplo de estrategias encaminadas a fortalecer el presupuesto de los SSJ, acuerdos con presidentes municipales en los que se brindaba atención al personal del ayuntamiento a cambio de apoyos económicos; convenios con instituciones como el IMSS para la atención de derechohabientes o bien sistemas de atención conjunta en localidades donde no se contaba con infraestructura médica o resultaba poco costeable mantenerla, a cambio de pago por servicio dado u otras compensaciones económicas; alianzas y convenios estratégicos con empresarios de la iniciativa privada que no se hubieran dado sin la descentralización de los SSJ.

\section{Tipo de decisión}

Existe consenso sobre el desconocimiento de la existencia de algún documento, normatividad o lineamiento interno de los SSJ, que oriente a los tomadores de decisiones sobre cómo se deben asignar o distribuir los recursos financieros. Al parecer, los cambios internos que se han presentado a partir de la descentralización en la forma de asignación de los recursos financieros en los SSJ no tienen un marco jurídico: no existe una ley específica en el estado, o normatividad propia de los SSJ, que implique la equidad y la protección financiera. Sin embargo, se dijo, es algo que está presente en la mente de quienes dirigen la institución y con base en eso se toman decisiones. Se piensa que los SSJ no pueden proponer un marco legal propio de estos cambios en el financiamiento y equidad por su ligadura tan estrecha con el nivel federal.

Se refirió que las regiones sanitarias cuentan con una guía administrativa para el ejercicio del gasto, en la que se incluyen las principales partidas que utiliza comúnmente el estado para explicitarles cuáles sí pueden ejercer y cuáles no, sirviendo además como una dinámica para hacer el control del gasto. Por otra parte, cuentan con un documento donde la DGP establece lineamientos y políticas globales claras sobre los porcentajes específicos que deben destinarse para el abasto de medicamentos, les fija topes para el gasto de Capítulo 5000 y establece gastos mínimos para mantenimiento.

En cuanto a los mecanismos para la asignación de los recursos financieros, a partir de la política descentralizadora se han presentado algunos cambios entre los que el personal entrevistado destaca los presentados en el cuadro I. Se pueden identificar, de hecho, dos escenarios completamente diferentes, que muestran un aparente éxito de la descentralización. En este orden de ideas, dividen la asignación de los recursos financieros en los SSJ en dos grandes bloques (figura 1). Por un lado, para las distintas direcciones generales y, por el otro, la asignación que se hace por centros de responsabilidad: regiones sanitarias y hospitales.

El personal entrevistado manifestó que no son muy claros los criterios utilizados para asignar los recursos financieros para las direcciones generales, ya que siguen basándose en los gastos históricos, siendo éstos el criterio fundamental.

Por ejemplo, nuestras direcciones generales en base a qué [...] criterios distribuimos el recurso del estado a las direcciones generales para su operación, y entonces nos vamos a históricos: ¿cuánto ejerciste el año pasado?, ¿el año antepasado?, pero sin saber exactamente, o sea nos hace falta una administración más específica de los que estamos poniendo el dinero y qué impacto estamos logrando para el siguiente año orientarlo, fortalecerlo o reorientarlo; o sea en algunas áreas no ha tenido gran cambio los criterios de asignación de recursos.

Por otro lado, se mantiene un monitoreo constante del ejercicio del gasto a lo largo del año para ver su conducta y detectar sobreejercicios o sobreahorros y, de esta manera, sobre la marcha hacer las transferencias internas necesarias para subsanar déficits y aprovechar ahorros. 


\section{Cambios en la ASIgnación de los ReCURSOS finANCIEROS POR REgIONES SANITARIAS Y hospitales}

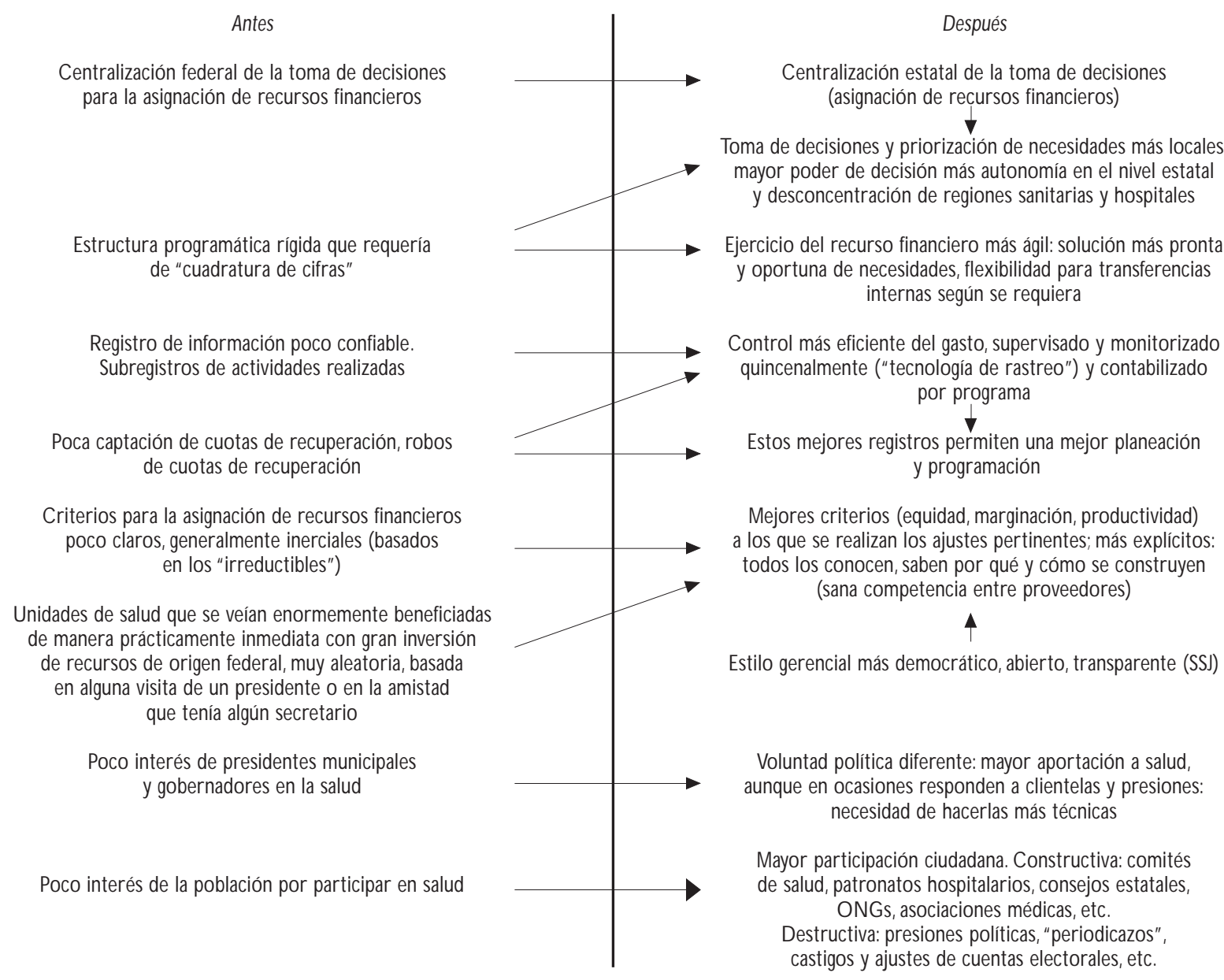

Las áreas identificadas como más prioritarias participan activamente negociando de forma directa con el secretario de Salud o con el director general de planeación para priorizar las necesidades y, en consecuencia, recibir las respectivas partidas presupuestales, mientras que a las demás no se les toma mucho en cuenta, situación que les genera un sentimiento de impotencia.

Desconozco, en primer lugar, el presupuesto general asignado a la Secretaría de Salud, asignado a mi Dirección General, asignado a mi Dirección de Área de la cual soy responsable. No hay suficiente información ni del dinero que llega, ni del control..., simplemente estamos a la deriva de quien me dice que ya no hay dinero. No hay dinero, pero veo que lo están invirtiendo en otras cosas, entonces ¿hay dinero o no hay dinero? o ¿para quién sí hay dinero o para quién no hay dinero?, no lo sé. Yo no dudo, ni con esto pongo en duda la capa- cidad, honestidad de quienes manejan el dinero, simplemente no lo sé.

Dentro de las direcciones generales, algunas áreas mencionan que toman mucho en cuenta las necesidades notificadas por el personal a su cargo, mismo que mediante programación anual establece sus requerimientos. Una vez autorizado el presupuesto, se hace la distribución al interior de la dirección de manera conjunta y participativa. Esta situación no prevalece en todas las direcciones.

En cuanto a la forma en que se asignan los recursos financieros por regiones sanitarias y hospitales se destacan, por un lado, algunos acuerdos-incentivos de gestión considerados como una fortaleza importante del estado de Jalisco. Un ejemplo es la cuota de recuperación que en años recientes ha incentivado a los administradores para incrementar su recaudación. En este sentido, la DGP ha establecido un porcentaje de 


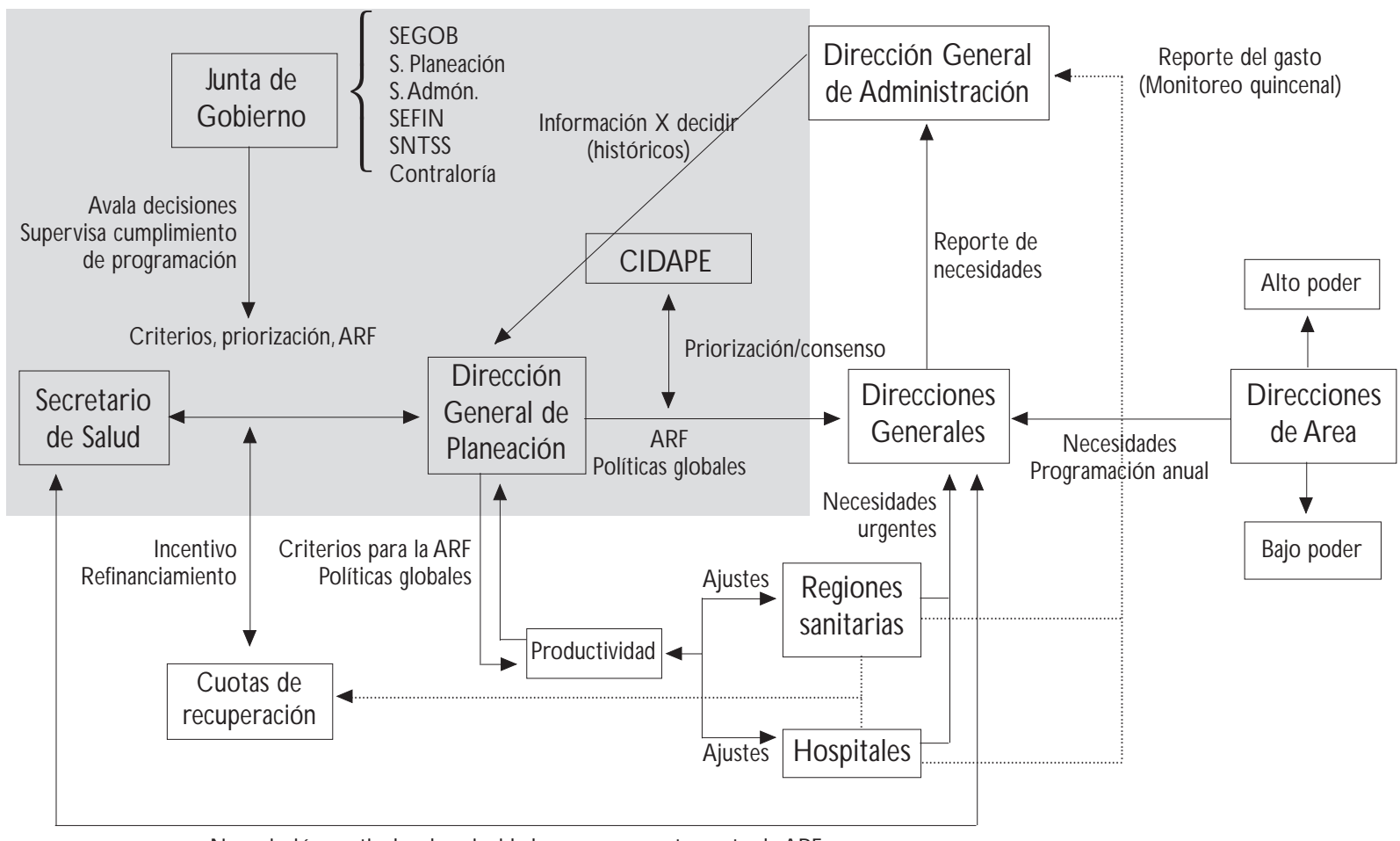

N egociación particular de prioridades y consecuentemente de ARF

SEG O G : Secretaría del Gobierno del Estado

SEFIN : Secretaría de Finanzas del Gobierno del Estado

SN TSS: Sindicato N acional de Trabajadores del Sector Salud

ARF:Asignación de Recursos Financieros

CIDAPE: Comité Interno de Administración, Planeación y Evaluación

Figura 1. Asignación de recursos financieros al interior de los Servicios de Salud, Jalisco

los ingresos con el que los centros de responsabilidad se pueden quedar, aunque sigue siendo esta área la que decide cómo y en qué gastar dichos recursos. Se trata de una especie de acuerdos "no escritos" de gestión.

Por otro lado, existen algunos criterios que se destacan en la asignación de recursos financieros a las regiones sanitarias y hospitales. Así, se afirmó que se da prioridad a la prevención sobre la curación con base en criterios implícitos de costo-efectividad. Otro criterio citado para decidir en qué gastar y cómo gastar los recursos financieros, por las mismas características de las instituciones de salud, son las urgencias epidemiológicas. En ocasiones, estas situaciones excepcionales los obligan a realizar transferencias internas extraodinarias para controlar y dar solución a dichas eventualidades. En este sentido, hicieron hincapié en el estrecho monitoreo que hacen del comportamiento epidemiológico de cada región a partir de los reportes estadísticos que se hacen y las supervisiones constantes por diferentes áreas que observan también la dinámica de trabajo, con el fin de ser alertados oportunamente $y$, en su caso, proponer correcciones y reasignaciones de recursos financieros con base en sus evaluaciones.

La administración actual refirió los tres objetivos que se propuso cumplir en cuanto a la distribución presupuestal: a) dar servicio de salud a toda la población; b) fortalecer la calidad de los servicios y c) premiar el esfuerzo fortaleciendo las unidades de responsabilidad con los insumos necesarios para brindar una atención de calidad. De esta forma, los SSJ adoptaron una metodología innovadora, tanto para la asignación de los recursos financieros destinados a regiones sanitarias como para aquellos destinados a los hospitales. Este modelo asigna un valor en Unidades Presupuestales de Asistencia Sanitaria (UPAS) para cada una de las actividades prioritarias que se realizan en regiones sanitarias y hospitales -como por ejemplo detecciones, consultas, pláticas, días estancia, entre otras-, de tal manera que los recursos financieros son asignados de acuerdo con el número de UPAS generadas por cada centro de responsabilidad -diferenciando las regiones sanitarias de los hospitales- según su productividad, 


\section{Poder, gRado y tIPO de deCISIÓn POR ORIGen Del ReCURSO}

\begin{tabular}{|c|c|c|c|c|}
\hline & \multirow{2}{*}{\multicolumn{3}{|c|}{ Servicios de salud Jalisco }} \\
\hline & & & & \\
\hline $\begin{array}{c}\text { Fuente de } \\
\text { financiamiento }\end{array}$ & Tipo de recurso & Poder de decisión & $\begin{array}{l}\text { Grado de } \\
\text { decisión }\end{array}$ & Tipo de decisión \\
\hline \multirow[b]{2}{*}{ SSA } & Ramo 12 & Limitado & $0 \%$ & Lineamientos y normatividad federal \\
\hline & Ramo 33 & "Alto", sujeto a Capítulo 1000 & "100\%" & $\begin{array}{l}\text { De } 80 \text { a } 93 \% \text { del Capítulo 1000; el resto, direcciones } \\
\text { generales: históricos; regiones sanitarias y hospitales. } \\
\text { Productividad, equidad, complejidad hospitalaria }\end{array}$ \\
\hline Entidad Federativa & $\begin{array}{l}\text { Aportación estatal } \\
\text { para salud }\end{array}$ & $\begin{array}{l}\text { Limitado, poca participación de los SS] } \\
\text { en la toma de decisiones }\end{array}$ & $i ?$ & $\begin{array}{l}\text { Etiquetado por el gobierno del estado para: antirretro- } \\
\text { virales, infraestructura de zonas marginadas, ambulan- } \\
\text { cias, consejos estatales }\end{array}$ \\
\hline \multirow{4}{*}{$\begin{array}{l}\text { Dependencias del } \\
\text { gobierno estatal }\end{array}$} & $\begin{array}{l}\text { Secretaría de Desa- } \\
\text { rrollo Urbano }\end{array}$ & $\begin{array}{l}\text { Moderado: solicitud de apoyo, de acuer- } \\
\text { do a las necesidades propias de los SS] }\end{array}$ & i? & $\begin{array}{l}\text { N ecesidades de remodelación o construcción de insta- } \\
\text { laciones, evaluadas y priorizadas por los SSJ bajo crite- } \\
\text { rios técnicos }\end{array}$ \\
\hline & DIF & $\begin{array}{l}\text { Moderado: solicitud de apoyo, trabajo } \\
\text { conjunto }\end{array}$ & i? & A poyo a programas de salud (Ej: cáncer de próstata) \\
\hline & COPLADE & $\begin{array}{l}\text { Moderado: solicitud de apoyo, trabajo } \\
\text { de convencimiento }\end{array}$ & i? & $\begin{array}{l}\text { N ecesidades específicas (por ejemplo, diseño, implemen- } \\
\text { tación y financiamiento de sistema de inventarios de } \\
\text { medicamentos) }\end{array}$ \\
\hline & IJAS & Limitado & i? & Bienes mostrencos y/o dinero producto de su venta \\
\hline Municipios & Ramo 33: FASSA & $\begin{array}{l}\text { Moderado: gestión de apoyo para ne- } \\
\text { cesidades específicas, trabajo conjunto } \\
\text { en la priorización de necesidades }\end{array}$ & i? & $\begin{array}{l}\text { Respuesta a presiones de la población: construcción de } \\
\text { centros de atención, equipamiento, salud rural, contra- } \\
\text { tación de personal eventual, etc. }\end{array}$ \\
\hline 0 tras instituciones & IMSS & $\begin{array}{l}\text { Moderado: acuerdos de atención con- } \\
\text { junta (atención primaria a la salud), su- } \\
\text { brogación de servicios }\end{array}$ & $100 \%$ & $\begin{array}{l}\text { Un porcentaje se queda en la región sanitaria para sus } \\
\text { necesidades específicas. El resto pasa a nivel central para } \\
\text { ser repartido }\end{array}$ \\
\hline $\begin{array}{l}\text { Agencias interna- } \\
\text { cionales }\end{array}$ & Donaciones & $\begin{array}{l}\text { Limitado para las donaciones en espe- } \\
\text { cie o que van destinadas para rubros } \\
\text { específicos }\end{array}$ & i? & $\begin{array}{l}\text { Ubicación de las donaciones en especie (equipo y recur- } \\
\text { so financiero) de acuerdo con necesidades específicas }\end{array}$ \\
\hline \multirow{4}{*}{ Privado } & $\begin{array}{l}\text { Empresas privadas } \\
\text { (Coca-C ola, Yakult, } \\
\text { Promomedios Radio, } \\
\text { etc.) }\end{array}$ & $\begin{array}{l}\text { Moderado: gestión de recursos para sa- } \\
\text { tisfacción de necesidades detectadas }\end{array}$ & i? & Solución de necesidades específicas \\
\hline & Laboratorios & $\begin{array}{l}\text { Moderado: solicitud de apoyos específi- } \\
\text { cos, gestión de financiamiento alterno }\end{array}$ & i? & $\begin{array}{l}\text { Ubicación de las donaciones en especie (medicamen- } \\
\text { tos) y capacitación específica del personal médico }\end{array}$ \\
\hline & $\begin{array}{l}\text { Cuotas de recupe- } \\
\text { ración }\end{array}$ & Alto & $100 \%$ & $\begin{array}{l}\text { Un porcentaje se queda en la región sanitaria para sus } \\
\text { necesidades específicas. El resto se envía al nivel cen- } \\
\text { tral estatal }\end{array}$ \\
\hline & $\begin{array}{l}\text { Comités de Salud, } \\
\text { patronatos hospita- } \\
\text { larios, } 0 \text { N Gs, etc. }\end{array}$ & $\begin{array}{l}\text { Moderado: solicitud expresa de apoyo } \\
\text { (financiamiento alterno y ahorro de re- } \\
\text { cursos) }\end{array}$ & i? & N ecesidades específicas de los centros de atención \\
\hline
\end{tabular}

haciendo algunos ajustes por tipo de complejidad -en el caso de los hospitales- y por vulnerabilidad y rezago de la población -para el caso de las regiones sanitarias- a la luz del criterio de equidad y justicia. Estos ajustes consisten en dar valor diferenciado en UPAS para la misma actividad, dependiendo del lugar donde se realiza. De esta manera, el valor de cada UPAS en un año específico resulta del número total de UPAS generadas por todos los centros de responsabilidad en el año inmediato anterior entre los recursos financieros de que se dispone para el año en cuestión.

En este sentido, a partir del establecimiento de este sistema y debido a las reducciones presupuestales experimentadas, se comenta la experiencia de algunas 
unidades a las que hubo que reducirles el presupuesto de 30 a $40 \%$ con respecto al año anterior.

Aquellos grandes hospitales y aquellas regiones más pobladas, que supuestamente tenían [...] los mayores presupuestos, resultó ser con que no, con que se estaban emparejando con otras que aparentemente [...] no tenían tanta gente para atender como población abierta pero que en realidad estaban dando tanto o más servicios que en las más pobladas.

Existe la percepción generalizada de que esta metodología para la asignación de recursos financieros sí ha funcionado y que es de gran utilidad, aunque también se acepta y reconoce la importancia de enriquecerla y mejorarla, esto es, de realizar los ajustes, actualizaciones y afinaciones en los puntos débiles que se han presentado sobre la marcha. Es un método flexible que permite seguir adecuando los criterios de acuerdo con las necesidades emergentes y en lo que demuestra tener más impacto. De manera indirecta, esta estrategia ha mejorado el subregistro de las intervenciones realizadas.

Desde mi punto de vista sí [ha funcionado esta metodología]. Pues sí, ya que se enfoca principalmente a indicadores o sobre población, como te comento de tu utilidad y no deja a un criterio libre de decir... cierta preferencia a ciertas áreas o cierta región, sino es de alguna manera en base a datos reales, a lo que son cifras, de números que son fríos, pero al final de cuentas es un parámetro para medir de forma pareja, equitativa a todas las unidades. Mi punto de vista creo que es: sí es adecuado.

Esta metodología parte de un principio sano y elemental: el que más trabaja, más consume; más insumos necesitará y, por ende, se deben asignar más recursos. El mensaje es claro: aquel que trabaje más, tendrá más dinero. En este sentido, se transita de prácticas poco objetivas, con ciertas preferencias a áreas o regiones, hacia una forma objetiva y equitativa de asignar los recursos financieros.

Anteriormente la forma de administrar los recursos era un poco atípica, de manera tal que había unidades de salud que se veían enormemente beneficiadas de una manera prácticamente inmediata con una súper inversión de recursos que venían de origen federal, pero pues era distribución de recursos muy aleatoria, muy específica, muy asignada, a veces basada en la visita de un presidente, a veces basada en la amistad que tenia algún secretario; en fin, yo creo que los criterios de dis- tribución del gasto de inversión ahora son, sin lugar a dudas, mucho mejores que los que había antes.

Las principales desventajas comentadas por el personal directivo tienen que ver con la preocupación de crear un círculo vicioso en el que la baja productividad sea penalizada con bajo presupuesto y el bajo presupuesto impida, a su vez, aumentar la productividad. Por otro lado, reconocen que no hay estudios de costos que les permitan saber cuánto se gasta por actividad en salud -y las variaciones por centro de atenciónque pudieran, hasta cierto punto, justificar y sustentar de mejor manera esta forma de asignar el dinero. Otro problema comentado es que sólo se incentiva la productividad, sin tomar en cuenta la calidad de las actividades realizadas - por la misma complejidad que esto implicaría-, situación que podría hacer que, por un lado, se incremente el número de actividades en salud realizadas "al vapor" y sin un contenido ideal y, por el otro, que exista un registro "viciado" de actividades no realizadas. Tampoco contempla el impacto en salud de las intervenciones realizadas, situación que les representa un reto a futuro.

\section{Discusión}

Se sabe que los estados que participaron en el primer esfuerzo de descentralización en la década de los 80 , cuyo alcance fue sólo una desconcentración administrativa, estuvieron mejor preparados, desde el punto de visto técnico y administrativo, para el proceso de descentralización llevado a cabo en $1996 \cdot{ }^{13-18}$ En este sentido, resultó particularmente importante determinar, a través del estudio de caso de los SSJ, las implicaciones de haber participado en esta primera fase de descentralización y la repercusión de esos 11 años que el estado ganó de experiencia sobre la administración de sus programas y la nueva relación con la Federación respecto de la asignación de los recursos financieros. Se pudo observar que el aumento de la capacidad técnica y la experiencia adquirida por el personal administrativo han desempeñado un papel importante en el empoderamiento de los SSJ y en el ejercicio de la autonomía de la institución.

Las restricciones establecidas en los contratos laborales del personal de base tienen grandes implicaciones sobre el poder de decisión de los SSJ, mismas que son consistentes con lo informado en trabajos previos. ${ }^{13-16,18}$ Debido a que el gasto en el Capítulo 1000 representa la mayor proporción del destino final de los recursos financieros de los SSJ, el hecho de carecer de poder real sobre la administración del personal impide que el estado tome control sobre una de las herra- 
mientas principales para lograr los cambios que se requieren en la provisión de los servicios, ${ }^{18}$ y limita la efectividad de la política descentralizadora. En este sentido, entre las implicaciones de que los SSJ no tengan control directo sobre la nómina, prestaciones y estímulos, y demás aspectos relacionados, está el grave crecimiento en el gasto del Capítulo 1000 en detrimento del gasto operativo y de inversión. Se pierde la esencia y razón de ser de la institución al robustecer la burocracia institucional a expensas del servicio a la comunidad, situación que pudiera no presentarse si se les otorgara poder legal y administrativo absoluto sobre la plantilla laboral. De ahí que la centralización del Capítulo 1000 siga siendo una gran restricción para los estados y un aspecto pendiente de descentralizar, lo cual coadyuvaría en el incremento de la calidad, la eficiencia y la productividad en los servicios provistos por los SSJ. A este respecto, el mismo personal entrevistado manifestó la necesidad de hacer frente a esta situación y solucionar lo referente a las condiciones generales de trabajo, así como los vicios, usos y costumbres institucionales.

Por otro lado, las inequidades laborales, producto de compensaciones federales asignadas a determinados programas, así como el equipamiento que conllevan, son otro claro ejemplo de las consecuencias de enviar el dinero etiquetado a los servicios estatales de salud. No sólo el estado es incapaz de manejar dichos recursos conforme a sus necesidades, sino que también les produce problemas y conflictos internos. En este orden de ideas, el Ramo 12 es otro punto pendiente de descentralizar.

Si bien a partir de la descentralización los SSJ han incrementado su poder de decisión, las decisiones generalmente responden a políticas y lineamientos de las máximas autoridades estatales, quienes pudieran estar condicionando o limitando tal poder. Detectar que la libertad para asignar los recursos financieros se va restringiendo conforme se baja en la escala de jerarquías (perfil del entrevistado) y encontrar que algunas personas con altos puestos directivos y posiciones estratégicas desconocen todo lo relacionado a la forma en como los SSJ asignan los recursos financieros por regiones sanitarias y hospitales, habla de una tendencia a la centralización estatal, especialmente en algunas áreas estratégicas, lo que va en contra del espíritu de la descentralización. También muestra la poca participación e interacción que tiene el personal directivo en cuanto al establecimiento de los criterios y formas de trabajar. En este sentido, se pudo apreciar, a partir del discurso de los informantes, poca claridad en los criterios utilizados para asignar los recursos financieros por direcciones generales y de área. De manera con- sistente con lo documentado en otros estudios, ${ }^{13-16,18}$ estas asignaciones se siguen haciendo bajo la tendencia histórica del gasto, por lo que no todas las áreas se ven beneficiadas en su programación presupuestal y no se hace manifiesto, al menos para todas ellas, por qué ni cómo se distribuyen los recursos financieros. Esta es una ventana de oportunidad en la que habrá que trabajar al interior del organismo para transparentar, enriquecer y hacer más participativos los procesos internos de asignación de recursos financieros, pues la frustración e inconformidad que esto genera para algunas áreas y el sentimiento de impotencia experimentado por aquellas que se ven como menos prioritarias, pueden convertirse en importante incentivo negativo.

A pesar de que el grado de decisión sea aparentemente bajo, lo más importante es cómo se decide sobre los muchos o pocos recursos sobre los que se tiene poder de decisión. El poder es cuestión de perspectivas: se puede pensar que $5 \%$ del monto total sobre el que se decide en los SSJ es una cifra muy reducida; sin embargo, no lo será tanto si se toma en cuenta que ese porcentaje corresponde a entre 74 y 223 millones de pesos anuales, del gasto de los SSJ en el periodo de 1995 a 2003. De ahí la importancia de decidir la mejor manera de asignar los recursos financieros equitativamente, a fin de que rindan el mejor fruto.

Por otra parte, se destaca el hecho de que se programa de acuerdo con los recursos disponibles y no con las necesidades reales, tanto de la población como de la misma institución. La aparente falta de recursos y la mala eficiencia en la asignación impiden el cumplimiento de las metas ideales, y como consecuencia hacen que el impacto en salud de las actividades de los SSJ sea menor. Lo anterior alerta sobre la necesidad de buscar fuentes alternas de financiamiento y ahorros institucionales que redunden en el logro de sus objetivos. A este respecto, se pudo observar que la decisión sobre la inversión de los recursos financieros se inclina a favor de las posibilidades de hacer algo más grande y no hacia donde realmente se necesita. De la lista de prioridades, si se encuentra financiamiento complementario para cumplir la necesidad cuatro, se opta por aceptarlo e invertirlo en eso, y no en la prioridad número uno. Aquí se le plantea un dilema a la institución: dejar pasar el dinero porque no atiende a su prioridad número uno, o aprovechar la oportunidad de obtener financiamiento aunque no sea para lo más prioritario. Empero, reconocer que los recursos no están en los lugares más adecuados e identificar la necesidad de realizar trabajo al interior de la institución para destinar mejor los recursos, y así acortar la brecha que existe entre las necesidades y los recursos disponibles, es ya un signo de crecimiento institucional. 
A partir de 2003 se ha logrado corregir la tendencia a la baja que experimentaba la recaudación de las cuotas de recuperación, lo que sin lugar a dudas muestra el éxito de dicho incentivo administrativo. No obstante, a pesar de que las regiones sanitarias y los hospitales retengan parte del dinero recaudado vía cuotas de recuperación, y aunque esta vía haya cobrado un papel significativo para el refinanciamiento de los SSJ, los administradores tienen que sujetarse a las especificaciones del nivel central estatal, que determina en qué sí se puede gastar y en qué no. Valdría la pena analizar más a profundidad la manera en como se establecen estos lineamientos y si responden o no a las necesidades locales, situación que se podría conocer documentando la experiencia del personal operativo.

Los nuevos criterios que se están utilizando en los SSJ para distribuir el dinero por regiones sanitarias y hospitales -a través de UPAS-, parecen favorecer una repartición más equitativa de los recursos. En este sentido, será importante monitorear la tendencia del gasto experimentada por las regiones sanitarias y los diferentes hospitales para evaluar si realmente se han modificado las tendencias presupuestales, qué tanto y si en verdad responden a una lógica de equidad. Estos cambios experimentados en la asignación de recursos financieros por regiones sanitarias y hospitales no han sido documentados en el estado ni llevados al plano normativo, legislativo, situación que concuerda con experiencias previas. ${ }^{13,15}$ Esta situación hace muy probable que variaciones en la actual dirigencia puedan condicionar o facilitar también cambios en dichos criterios, lo que propiciaría no dar continuidad a dicha metodología y, por tanto, no poder evaluar su impacto sobre el funcionamiento de las unidades, el desempeño de los proveedores ni, finalmente, sobre la salud de la población. Además, la gestión de recursos por parte de los tomadores de decisión es un complemento importante de los criterios de asignación, tendientes a completar metas y acciones. ${ }^{15}$ El rol que han jugado, tanto el director general como su equipo de trabajo, para la implantación de dichos cambios, ha sido significativo y fundamental. Sin embargo, el hecho de que estos cambios dependan de una sola persona o de un grupo de trabajo determinado y de sus habilidades como gerentes-gestores, fomenta que los OPD sean manejados prácticamente como negocios particulares; de ahí que el concepto de servicio civil de carrera cobre gran relevancia. Al parecer, en los SSJ no existe una estructura organizacional tal que facilite y oriente la toma de decisiones con esquemas más participativos, para lo que se requiere modernizar la sistematización y automatización del capital humano y de la búsqueda de profesionalización, lo que pudiera lograr el crecimiento institucional y la continuidad de los proyectos, a fin de que la forma de trabajar de la institución no dependa sólo de una persona o un equipo de trabajo, sino que se convierta en una visión institucional a largo plazo.

Dentro de la lógica de esta metodología se identifican dos dimensiones de equidad que la justifican. Por un lado, la equidad institucional, la cual se basa en una asignación de recursos bajo criterios claros, explícitos - productividad, nivel de complejidad y tipo de población- $\mathrm{y}$ aplicables a todos, que fomenta la competencia justa entre proveedores. Por otro lado, la equidad poblacional, que otorga mayor asignación de recursos para los grupos de población -regiones sanitarias- que más lo necesitan, léase vulnerables, marginados y rezagados. En este sentido, se busca la justicia financiera o equidad en el financiamiento. Sin embargo, los supuestos presentados en el cuadro III son situaciones potencialmente riesgosas, pues de no cumplirse, el sistema está diseñado para introducir a las unidades de responsabilidad en un círculo vicioso: poca productividad, poco recurso financiero asignado, poca productividad. Asimismo, habrá que tener especial cuidado en no desatender a las regiones menos vulnerables en un intento por compensar a las más marginadas. Existe controversia entre el mismo personal en relación con los criterios o las bases sobre las que se decidió ponderar -de la zona rural alejada y marginada a la urbana cercana no marginada- y si no se estarían sobreponderando dichas asignaciones. En el afán de buscar la equidad de la población más alejada del centro, se puede propiciar una inequidad que afecte a la población urbana menos favorecida.

El esfuerzo estatal de años recientes, con incrementos referidos como importantes en su aportación a salud, bien podría ser considerado como un éxito de la política descentralizadora, al menos en este contexto particular, en el sentido de hacer que las entidades asuman su responsabilidad en salud e inviertan mayores recursos. No obstante, se rescata de este estudio el problema de que la aportación estatal no siempre se dirige hacia necesidades técnicamente detectadas y priorizadas y que se desconocen los criterios que utiliza el gobierno del estado para asignar los recursos financieros que destina a salud. Si bien se sabe que los recursos están etiquetados para atender las áreas marginadas, financiar algunos consejos estatales de salud, adquirir ambulancias para todos los municipios, así como para adquirir antirretrovirales y financiar el hospital psiquiátrico, no se sabe a ciencia cierta por qué se atienden estas necesidades en salud y no otras, y con qué fundamento se eligieron estos problemas. Es recomendable indagar con mayor profundidad los criterios que utilizó el estado para decidir cómo y en qué 


\section{Cuadro III SupUestos IMPLícitos de LA ASIGNACIÓN DE RECURSOS FINANCIEROS POR REGIONES SANITARIAS $Y$ HOSPITALES EN LOS SERVICIOS DE SALUD DEL ESTADO DE JALISCO}

- Los gastos de los centros de responsabilidad por las actividades de salud son los mismos para regiones con igual grado de marginación 0 vulnerabilidad.

- La calidad no disminuye al aumentar la productividad.

- La baja productividad es debido a deficiencias del proveedor y no se debe a barreras de acceso y problemas de utilización y cobertura 0 bien a baja calidad técnica en la oferta.

- Un estímulo negativo, como disminuir el presupuesto asignado a una región sanitaria u hospital, es la mejor manera para estimular al administrador a corregir fallas.

- Los gradientes y ponderaciones realizados, para compensar la complejidad hospitalaria y las características po blacionales, son los más adecuados.

gastar dichos recursos y en qué medida las agendas de gobierno responden a presiones sociales de grupos específicos y a políticas y asignaciones electorales ${ }^{*, 19}$ poco efectivas en salud. En este sentido, la aportación municipal en salud, que se ha incrementado recientemente, también pudiera responder a esta lógica. Existe el riesgo de que los servicios estatales de salud se vuelvan una herramienta política que convierta a la salud en arena de intercambio político entre el estado y la sociedad. ${ }^{20}$

Finalmente, la experiencia notificada a partir de este estudio de caso permitirá la retroalimentación a los demás servicios estatales de salud, aportando buenas ideas, con base en resultados satisfactorios, o alertar a partir de resultados poco satisfactorios, además de servir como punto de comparación para otros estudios de caso. Aquí destaca la importancia de dar continuidad a estos estudios de caso para observar el impacto del nuevo Sistema de Protección Social en Salud sobre la asignación de los recursos financieros en los estados, tema que no aborda el presente estudio.

\footnotetext{
* Aquí se da un sentido más amplio a las políticas electorales. Además de incluir el concepto de "votante movilizado", mismo que define Wayne A. Cornelius como el votante cuya participación electoral (asistencia) y/o elección de candidatos es inducida por la compra -intercambio de beneficios materiales y servicios por votos- y la coacción del voto -condicionamiento de la distribución de algún servicio o beneficio gubernamental al voto por un partido o candidato específico-; también comprende el intento de sacar ventaja electoral mediante la canalización de fondos públicos hacia sus principales o potenciales electores. Se trata de estrategias tradicionalmente realizadas con recursos de los programas de lucha contra la pobreza manipulados por los gobernadores de estado o presidentes municipales (referencia 19).
}

\section{Referencias}

1. C astaño R. Medicina, ética y reformas a la salud: hacia un nuevo contrato social con la profesión médica. 2a edición. Bogotá: ECOE Ediciones, 2000: 186.

2. Arredondo A. Indicadores de eficiencia para la asignación de recursos en salud. Rev Med Chile 1999; 127: 856-961.

3. Fundación Mexicana para la Salud (FUN SALUD). 0 bservatorio de la salud: necesidades, servicios, políticas. C apítulos 4, 5, 6, 11, 16. México D. F.: FUN SALUD, 1997:119-193; 255-274; 359-378.

4. Arredondo A. Equity and financing indicators in the context of decentralization: Political actors and strategies for health sector reform (phase 2) [protocolo de investigación]. México: Instituto $\mathrm{N}$ acional de Salud Pública, 2003: 38.

5. Secretaría de Salud. D escentralización de los servicios de salud: el desafío de la diversidad. En:C uadernos de Salud 4. México D.F.: SSA, 1994: 49.

6. So berón-A cevedo G, Martínez-N arváez G . La descentralización de los servicios de salud en México en la década de los ochenta. Salud Publica Mex 1996; 38: 371-378.

7. Historia. Cambios legales de la Secretaría de Salud y Bienestar Social en el Estado de Jalisco. [consultado 2004 feb 15]. D isponible en: http:// ssj.jalisco.gob.mx/index1.html

8. Poder Ejecutivo Federal. Programa de Reforma del Sector Salud, México, D.F.: PEF, 1996.

9. Moreno JC. La descentralización del gasto en salud en México: una revisión de sus criterios de asignación [disertación]. 2001. [consultado 2004, 20 ene ]. Disponible en: http://www.presupuestocide.org/ documentos/salud/DT\%2095.pdf

10. Diario 0 ficial de la Federación. A cuerdo $\mathrm{N}$ acional para la Descentralización. México: D.F.: Miércoles 25 de septiembre de 1996. 11. Diario 0 ficial de la Federación. D escentralización del O PD SSJ. Martes 6 de mayo de 1997.

12. Gobierno de la República Mexicana. Q uinto Informe de Gobierno; México, D.F.: 1999.

13. N igenda G,Valdez R, Ávila L, Ruiz JA. Descentralización y programas de salud reproductiva. México D.F.: Fundación Mexicana para la Salud/ Instituto N acional de Salud Pública, 2002:199.

14. U galde A, Homedes $N$. D escentralización del sector salud en A mérica Latina. Gac Sanit 2002; 16(1): 18-29.

15. Arredondo A. El financiamiento a partir de la descentralización del sistema de salud en México: cambios, tendencias y evidencias, 1990-2000. Cuernavaca, México: Instituto N acional de Salud Pública, 2002: 150. 16. Arredondo A, Parada I. Health financing changes in the context of health care decentralization:The case of three Latin American countries. J Public Health 2000; 34(5): 449-60.

17. A dams 0 , Shengelia B, Stilwell B, Larizgo itia I, Issakov A, K wankam SY, et al. Provision of personal and non-personal health services: Proposal for monitoring. En: Murray CJL, Evans D B. Health systems performance assessment: D ebates, Methods and Empiricism. Ginebra: 0 rganización Mundial de la Salud, 2003: 235-249.

18. Merino G. Descentralización del sistema de salud en el contexto del federalismo. En: Knaul F, N igenda G, eds. Caleidoscopio de la salud: de la investigación a las políticas y de las políticas a la acción. México D. F.: FUN SALUD, 2003: 195-207.

19. W ayne AC . La eficacia de la compra y coacción del voto en las elecciones mexicanas de 2000. Perf Latinoam, 2002; 20: 11-31. 20. Arredondo A. Federalismo y políticas de salud: descentralización y relaciones intergubernamentales desde una perspectiva comparada. México D.F.: Foro de Federaciones-SSA, 2003: 201. 